\title{
The effects of random whole-body-vibration on motor symptoms in Parkinson's disease
}

\author{
Christian T. Haas ${ }^{\mathrm{a}, *}$, Stephan Turbanski ${ }^{\mathrm{a}}$, Kirn Kessler ${ }^{\mathrm{b}}$ and Dietmar Schmidtbleicher ${ }^{\mathrm{a}}$

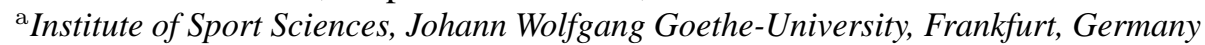 \\ ${ }^{\mathrm{b}}$ Department of Neurology, Johann Wolfgang Goethe-University, Frankfurt, Germany
}

\begin{abstract}
It is well known that applying vibrations to men influences multiple physiological functions. The authors analysed post effects of whole-body-vibration (WBV) on motor symptoms in Parkinson's disease (PD). Sixty-eight persons with PD were randomly subdivided into one experimental and one control group. Motor symptoms were assessed by the UPDRS (Unified Parkinson's Disease Rating Scale) motor score. A cross-over design was used to control treatment effects. The treatment consisted of 5 series of whole-body-vibration taking 60 seconds each. On average a highly significant $(p<0.01)$ improvement of $16.8 \%$ in the UPDRS motor score was found in the treatment group. Only marginal changes $(p>0.05)$ were found in the control group. The cross-over procedure showed comparable treatment effects (14.7\% improvement after treatment). With respect to different symptom clusters only small changes were found in limb akinesia and cranial symptoms. By contrast, tremor and rigidity scores were improved by $25 \%$ and $24 \%$, respectively.

According to the structure of symptom changes it is unlikely that these effects are explainable on peripheral sensory level, exclusively. With respect to the findings of other studies one can speculate about changes in activation of the supplementary motor area and in neurotransmitter functions.
\end{abstract}

Keywords: Random whole-body-vibration, Parkinson, UPDRS, motor-control

\section{Introduction}

Beginning with the animal and human experiments of Matthews or Hagbarth and Eklund in the 1960s it is well described that vibration applied to the musculartendon system can elicit a reflex muscle contraction [27, 47]. Apart from this phenomenon it became evident in numerous studies that vibratory stimuli modify a wide variety of physiological functions e.g. brain activation, hormone concentrations or neurotransmitter releases [3,4,6,49,51,52]. Moreover it is known that different vibration characteristics (e.g. whole-body vs. local) and parameters (e.g. frequency, amplitude) influence these effects strongly [20,25,26]. As propriocep-

\footnotetext{
* Address for correspondence: Dr. Christian T. Haas, Johann Wolfgang Goethe-University Frankfurt/Main, Institute of Sport Sciences, Ginnheimer Landstrasse 39, 60487 Frankfurt, Germany. Tel.: +49 69798245 23; Fax: +49 69798245 74; E-mail: c.haas@sport.unifrankfurt.de.
}

tion is an important component of motor control the influence of vibrations were analysed in a huge number of studies. Regardless of whether vibrations were applied locally to various muscles or to the whole-body a prominent influence on motor control became evident $[7,14,22,26,29-31,70-73]$. During tracking tests strong over- or undershooting errors were found [39,70, 71]. In upright stance vibration stimuli led to involuntary anterior-posterior whole-body sways [29,40-42]. Wiezbicka and co-workers found that these sways occur not only during the treatment but also some minutes afterwards [73]. Other researchers showed that vibrations applied to various muscles during walking modify the velocity and the direction of gait $[7,30,31,72]$. The physiological background of this phenomenon mostly described as 'kinaesthetic illusion' - consists in a misinterpretation of the vibratory stimulus due to its artificial character.

In contrast to the results found in healthy subjects vibrations seem to have a lower impact on coordination in 
Parkinson's disease patients. Rickards and Cody found significant lower undershooting errors in PD patients compared to healthy subjects during voluntary wrist extension movements and vibration transfer to the flexor carpi radialis [57]. Khudados et al. showed comparable low impacts of vibrations on tracking performance in PD compared to age matched controls [43]. It is argued that these differences result from a pathologically modified proprioception in PD. However, these vibration stimuli still influenced motor control of PD patients. Anyway the literature shows that the effect of vibration depend on the vibration parameters. Thus several experiments showed that vibration frequencies of more than $20 \mathrm{~Hz}$ are necessary to generate kinaesthetic illusions [14,50,67]. Furthermore Ivanenko et al. found that kinaesthetic illusions are influenced in upright stance by the support stability [29]. Thus instable support reduced the degree of illusion.

In consequence, it is likely that a low frequent wholebody-vibration connected with instable support would not generate negative effects. Furthermore a couple of experiments in the field of biomechanics and exercise physiology showed that vibratory stimuli can also improve neuromuscular performance ([26] for review). In PD acute reductions in tremor were found using local muscle vibration [35]. In addition, numerous PD patients report that symptoms are markedly reduced in vibratory situations e.g. train travelling. The guiding aim of the study at hand was therefore to analyse the effects of whole-body-vibration on motor control in PD patients.

\section{Materials and methods}

\subsection{Subjects}

Sixty-eight patients (15 female, 53 male) diagnosed with idiopathic Parkinson's disease participated in the study. The mean age $( \pm \mathrm{SD})$ was $65.0 \pm 7.8$ years, and the duration of the disease was $5.9 \pm 4.6$ years. Diagnosis of PD was established by the primary care neurologist on the basis of unilateral onset, asymmetric motor symptoms, symptom relief by dopaminergic treatment, and absence of atypical clinical signs such as severe orthostatic hypotension, cerebellar or pyramidal signs, early falls or gaze abnormalities, and normal brain imaging (computed tomography or magnetic resonance imaging). Patients with dementia or other diseases impairing gait, stance or coordination (e.g. neuropathy, muscle or joint disease) were not admitted to the study and all subjects had to be able to stand unsupported to participate.

Prior to study entry the severity of motor symptoms was assessed by the Hoehn \& Yahr scale (H\&Y) as well as the motor part of the Unified Parkinson's disease rating scale (UPDRS motor score). H\&Y reached from stage II to IV, the average UPDRS motor score (off medication) was $29.9 \pm 11.9$.

In 63 subjects normal daily medication included LDOPA (325 mg \pm 122 per day) and dopamine agonists, 13 subjects were treated only with dopamine agonists.

All tests and treatments were performed between 11:00 am and 3:00 pm. To exclude the influence of medication all patients were withdrawn from L-DOPA over night ( $>12$ hours). Subjects were not withdrawn from dopamine agonists, by the reason of longer average dissolution times (up to 110 hours).

All subjects participated in this study with their informed consent.

\subsection{Experimental setup}

The experimental procedure is based on three successive test sessions. In each test session, two patients were studied in a parallel cross-over design (see Fig. 1) and randomly assigned to one of two treatment groups. One group (A) received whole-body-vibration (WBV) as specified below first, followed by a resting phase. The other group (B) had the resting period first and was treated thereafter. Scoring (UPDRS motor score) was performed 3 times in each patient, i.e. at baseline (UPDRS 1), after the treatment session for group A (UPDRS 2) and after the treatment session for group B (UPDRS 3). Scoring was always carried out by the same examiner, blinded for the treatment status of the patient.

\subsection{Whole-body-vibration (WBV)}

For the treatment, the patient stood with shoes and with the knees slightly bent on a platform allowing separate and unsynchronised multidimensional WBV applied to both feet (ZEPTOR ${ }^{\circledR}$-med system, Scisens, Germany). WBV was administered in 5 series lasting one minute each with one-minute pause between each series. With respect to information selection processes a random vibration characteristic was chosen. Based on basic analyses it is known that variable stimuli provide greater potential to improve adaptation processes of the sensory motor system [26,60]. Furthermore these stimuli cannot generate resonance catastrophes or kinaesthetic illusions $[22,25,26]$. The mean frequency of the vibration was $6 \mathrm{~Hz}( \pm 1 \mathrm{~Hz} / \mathrm{s})$, the amplitude was $3 \mathrm{~mm}$. 


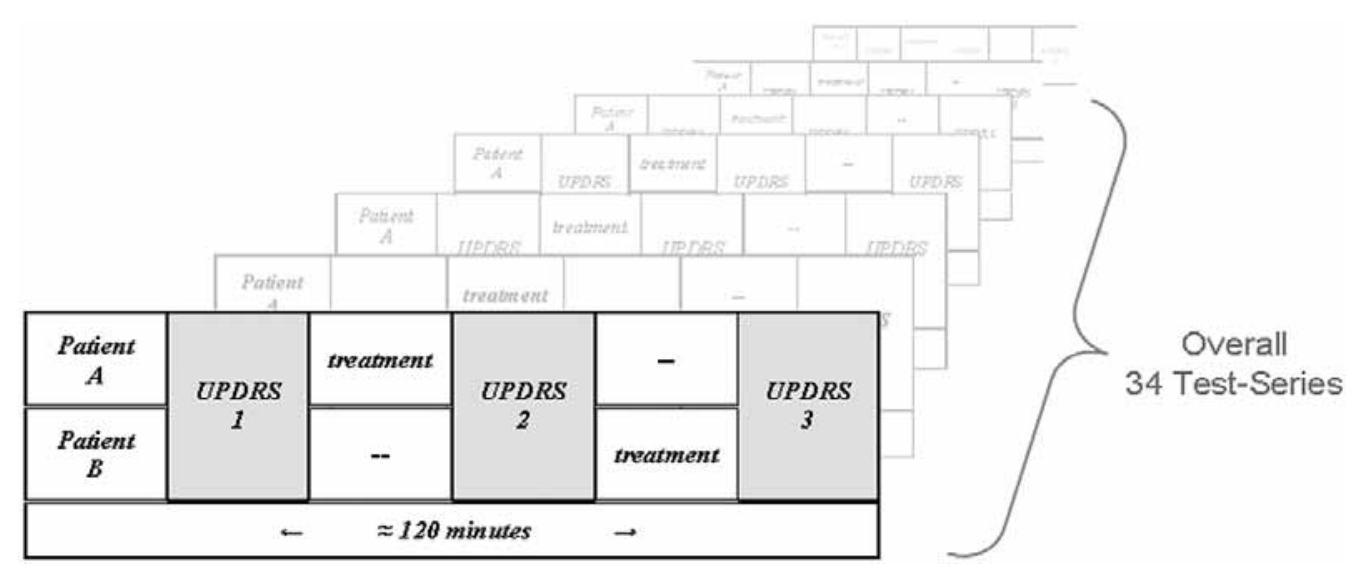

Fig. 1. Experimental setup.

\subsection{Data analysis and statistics}

The primary outcome measure was the difference between the UPDRS motor scores before and after WBV treatment. Secondary outcome measures were scores in subscales of the UPDRS reflecting tremor (items 20 and 21 , range $0-28$ ), rigidity (item 22, range $0-$ 20), bradykinesia (items 23-26 and 31, range 0-32), gait and posture (items 27-30, range 0-20) and cranial symptoms (items 18 and 19, range 0-8). Initially conventional explorative statistics were used to calculate mean and SD values and to prove data distributions. Parametric statistics were used in this experiment since they are more powerful than non-parametric methods and Monte Carlo simulations showed stable results for this kind of research designs [5]. Due to the parallel cross-over design a two ways ANOVA for repeated measures was calculated to compare treatment effects with baseline UPDRS of both groups (group A: first treatment, then rest; group B: first rest, then treatment). When needed Post-hoc analyses (Scheffé) were performed in a further step. As the disability range of the subjects was characterized by a wide variety patients were subdivided into five conjunct groups based on symptom patterns. Thus a subject was assigned to one subgroup if scored 2 or higher in one or more items (e.g. item 22: rigidity neck) of the symptom cluster (e.g. symptom cluster: rigidity). In each cluster prepost differences were proved using 1-way ANOVA for repeated measures. Pearson correlations were applied to identify possible interactions between treatment effects and further data like duration of disease, age or medication.

\section{Results}

All patients tolerated the procedure well and without reporting immediate or delayed adverse effects such as dizziness, kinaesthetic illusions, discomfort, pain or dyskinesias. Both groups do not differ significantly in age (group A: 64.1 \pm 7.0 , group B $65.8 \pm 8.3$ ), pharmaceutical treatment (group A: $319 \pm 105$, group $\mathrm{B}: 333 \pm 125$ ) or duration of the disease (group A: 5.8 \pm 4.5 , group B: $6.0 \pm 4.8$ ).

Figure 2 shows changes in the UPDRS motor scores during the experiment. In both groups, the UPDRS motor score was significantly $(p<0.01)$ reduced following WBV treatment on average by 5.2 (group A) and 4.8 (group B) score points corresponding to reductions of $16.8 \%$ and $14.7 \%$, respectively. Both groups showed no significant changes in UPDRS motor score following the control condition (waiting).

In order to identify the physiological structure of the WBV treatment different symptom clusters were analysed (Fig. 3). The highest improvements were found in tremor and rigidity (25\% and 24\%). Gait and posture items show $15 \%$ improvement on average. Bradykinesia scores were reduced by $12 \%$ on average, whether no changes were found in cranial symptoms. Score changes of all clusters were highly significant $(p<0.01)$ except the cranial symptom cluster.

In all groups low and insignificant correlations between UPDRS improvements and the initial UPDRS score, age, duration of disease or medication were found.

\section{Discussion}

As the treatment was connected each time with significant improvements in the UPDRS motor score while 


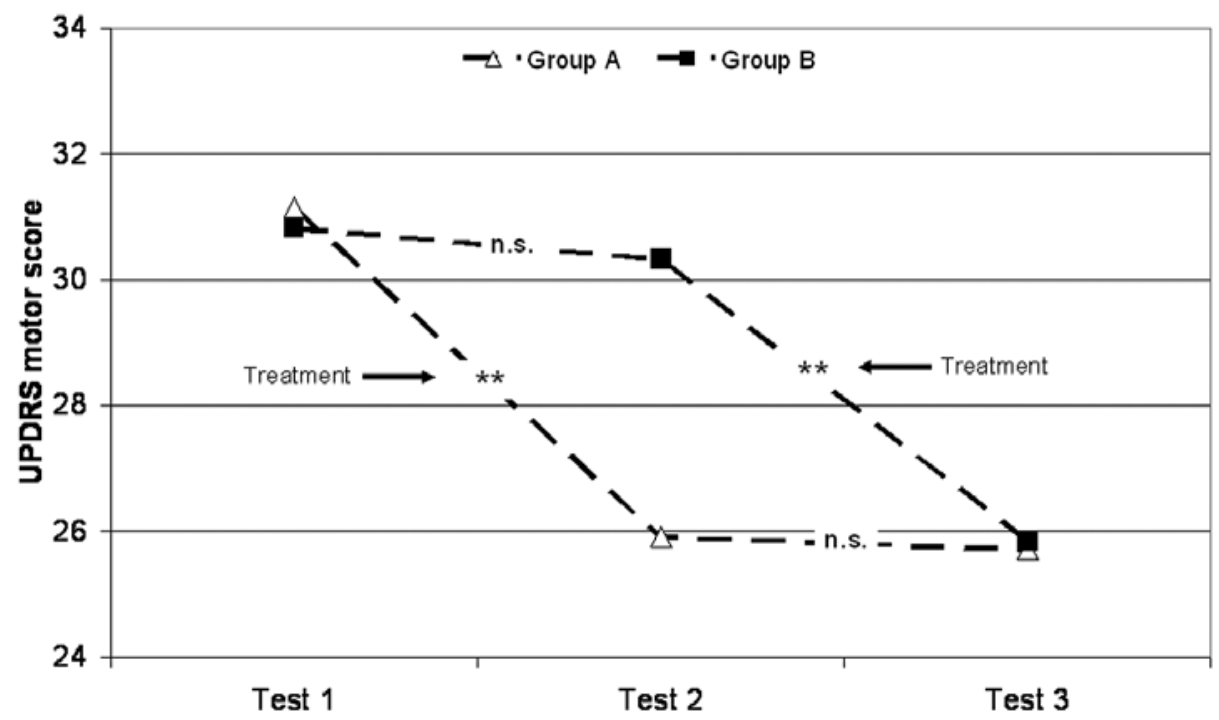

Fig. 2. Changes of the UPDRS motor score during test- and treatment-procedure (Group A: first treatment, then rest; Group B: first rest, then treatment).

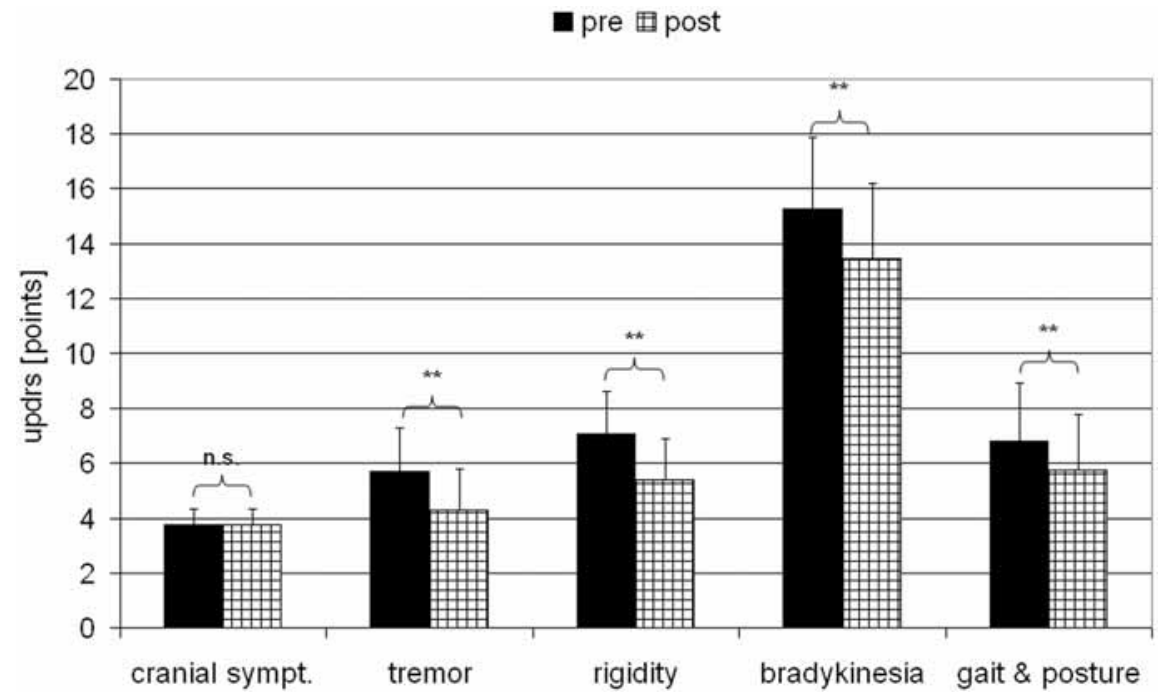

Fig. 3. UPDRS motor scores pre and post treatment in five symptom cluster.

the control condition led to small, insignificant changes only, one has to conclude that the treatment has beneficial effects on PD motor symptoms. Nevertheless, short term symptomatic fluctuations and spontaneous pharmaceutical aftereffects can not be excluded in all subjects. However, with respect to relatively large sample sizes it is unlikely that these fluctuations manipulate the main results of the study. Furthermore placebo effects might bias the results. Thus in a couple of studies it was found that placebo treatments can influence PD symptoms [15,16,20,21]. Goetz and co-workers analysed placebo associated symptom changes in early PD. On one hand they found that motor control improvements occurred in all clinical domains, on the other hand stronger improvements became evident in bradykinesia and rigidity than in tremor or gait and balance [20,21]. In contrast to these findings the study at hand shows a different effect pattern i.e. relatively strong improvements in tremor but small changes in bradykinesia. Furthermore biomechanical experiments - which enable a comprehensive and valid assessment of different components of postural control - showed that the WBV 
treatment leads to significant improvements in postural control $[23,24]$. The literature shows no comparable effect in postural control neither as a result of medication nor of placebo treatments $[33,48]$. Therefore, it is unlikely that the effects of our study are placebo associated totally and one has to look for other mechanisms. In this context it is worthy to emphasise time related stability of effects. 120 minutes after the first treatment the UPDRS score of group A (test 3) shows no return to baseline but still a small reduction. Consequently physiological functions should be discussed that provide potential to explain also middle-term effects.

Numerous experiments showed that the effects of vibratory stimuli are not limited to a single physiological system. Therefore, various functions have to be taken into account. The study at hand found symptom changes in the lower as well as in the upper body. Effects in the lower body might be explainable by adaptations on peripheral nerval level. The influence of vibrations on sensory function and motor control are described to a large extent in literature [7,14,22,26,29-31, 70-73]. In contrast to these explanations effects in the upper body might only be explainable by modifications of cortical or subcortical functions. A few animal and human experiments found that WBV can influence the concentration of several neurotransmitters $[3,4,6,49$, 51,52]. As the release functions are highly non-linear it is difficult to identify a specific stimulus-reaction relation. However, Schulz found that the sensitivity of dopamine neurons is associated with the predictability of a stimulus $[62,63]$. In a couple of experiments he showed that unpredictable and novel situations lead to phasic dopamine releases. In a further step reactions within the dopaminergic system are strongly connected with reinforcement and motor learning [11,62, 63]. By the reason of random vibrations we used in this study unpredictable stimuli are given which might result in enhanced dopamine releases. However, the duration of this biochemical effect is not clear but one can speculate that even a short additional dopamine release might provide a basis to reset pathologically activated brain circuits. Thus a couple of studies working with transcranial-magnetic-stimulation found that these stimuli led to additional dopamine releases, which have therapeutic effects $[44,45,54,65,66]$.

Besides biochemical consequences described above one can speculate in a further step about changes of brain activation. Even if current cortical and subcortical models still present a simplistic view there are evidences for strong connections between the basalganglia and the cerebral cortex $[1,62]$. It is well described that the supplementary motor area (SMA) is characterized by less activation in PD compared to healthy subjects $[9,11,32,34,53,56,58,59]$. However, the degree of pathological activation depends on the motor task. In contrast to a free selection of movement initiation external cues lead to less or no differences in SMA activation between PD and healthy subjects. Accordingly the vibration treatment provides external cues and therefore might normalizes SMA activation. Based on the results of other training experiments similar explanations can be found. The authors argue that gait training using a treadmill provides external cues and thereby internal cueing problems of PD patients are compensated and motor control is improved $[10,69]$.

With respect to vibration stimuli and brain activation, a recent study compared sinus waves with random oscillations [53]. It was shown that unpredictable random stimuli led to greater SMA activation than predictable oscillations did. It is well described that SMA is important for generating and controlling complex movements; however, it is unclear to which extent SMA activation generated during the treatment can influence post treatment motor control [32]. Apart from SMA functions Nelson and colleagues showed that an unpredictable treatment leads to relatively strong activations of prefrontal areas [53]. On one hand, these structures are known to be important for new learning or non routine decision, on the other hand, they are less active in PD which may explain learning and information selection deficits in PD [4,36,37,53,58,59].

Besides the underlying neurophysiological mechanisms of the treatment some practical and therapeutic aspects should be discussed. Principally it is difficult to speculate about long term effects of the treatment since both WBV training as well as medication influence motor symptoms, and furthermore PD is characterised by a high heterogeneity [55]. However, a few longterm observations showed that the treatment effects described above are no single phenomena. According to the problems of chronic L-Dopa treatment like psychiatric complications, dyskinesia, on-off fluctuations, and additional risk of cell death the vibration treatment might be effective in early PD stages to postpone the start of L-Dopa $[17,18,46]$. In this context some animal experiments found that physical activity has protective effects on striatal dopamine metabolism [12,13, 68]. Unilateral 6-OHDA infusions led in rats to a strong loss of DA (dopamine) and DOPAC (dihydroxyphenylacetic acid). In rats that were forced to use parkinsonian limbs DA and DOPAC loss was strongly reduced which was connected with markedly reduced parkinso- 
nian symptoms. Since an increase in neurotrophic factor protein in the striatum was found the authors speculate about a functional connection between exercise and protection of nerve cells. Other studies support this hypothesis $[12,13,68]$. With respect to the findings of Chen et al. muscle spindle activity regulates neurothrophic factors [12]. As WBV treatment results in strong spindle activity one can speculate that these stimuli play an important role in surviving process of nerval structures.

Another consideration refers to postural control and the importance for quality of life $[28,38,61]$. In later stages of PD gait impairment and postural instability are very common but pharmaceutical treatments are lowly beneficial or might even worsen these symptoms $[8,19$, $38,55]$. Biomechanical analyses showed that the treatment improves postural control of PD patients, which might ensure mobility and quality of life [23,24]. Finally, vibration training should not be regarded as an exclusive treatment but it could support conventional PD therapy.

\section{References}

[1] G.E. Alexander, M.D. Crutcher and M.R. DeLong, Basal ganglia - thalamocortical circuits: parallel substrates for motor, oculomotor, prefrontal and limbic functions, Prog Brain Res 85 (1990), 119-146.

[2] M. Ariizumi and A. Okada, Effect of whole body vibration on the rat brain content of serotonin and plasma corticosterone, Eur J Appl Physiol Occup Physiol 52 (1983), 15-19.

[3] M. Ariizumi and A. Okada, Effect of whole body vibration on biogenic amines in rat brain, Br J Ind Med 42 (1985), 133-136.

[4] H. Bocker, A. Ceballos-Baumann, P. Bartenstein, A. Weindl, H.R. Siebener, T. Fassbender, F. Munz, M. Schwaiger and B. Conrad, Sensory Processing in Parkinson's and Huntington's disease: Investigations with $3 \mathrm{Dh}_{2}$ 15O-PET, Brain 122 (1999), 1651-1665.

[5] J. Bortz, G.A. Lienert and K. Boehnke, Verteilungsfreie Methoden in der Biostatistik, Berlin, Springer 2nd ed, 2000.

[6] C. Bosco, M. Iacovelli, O. Tsarpela, M. Cardinale, M. Bonifazi, J. Tihanyi, M. Viru, A. de Lorenzo and A. Viru, Hormonal responses to whole-body vibration in men, Eur J Appl Physiol 81 (2000), 449-454.

[7] M. Bove, M. Diverio, T. Pozzo and M. Schieppati, Neck muscle vibration disrupts steering of locomotion, J Appl Physiol 91 (2001), 581-588.

[8] H.M. Bronte-Stewart, A.Y. Minn, K. Rodrigues, E.L. Buckley and L.M. Nashner, Postural instability in idiopathic Parkinson's disease: the role of medication and unilateral pallidotomy, Brain 125 (2002), 2100-2114.

[9] D.J. Brooks, Functional imaging studies on dopamine and motor control, J Neural Trans 101 (2001), 1283-1298.

[10] T.T. Capato, E.S. Takata, M.C. Moura, M.C. Fornari, E.R. Barbosa and M.E. Piemonte, Rapid gait improvement in Patients with Parkinson's disease through training based on movements guided by rhythmic cues, Mov Disord 19(Suppl. 9) (2004), 210,590 .
[11] M.J. Catalan, K. Ishii, M. Honda, A. Samii and M. Hallett, A PET study of sequential finger movements of varying length in patients with Parkinson's disease, Brain 122 (1999), 483-495.

[12] H.-H. Chen, W.G. Toutelotte and E. Frank, Muscle SpindleDerived Neurotrophin 3 regulates synaptic connectivity between Muscle Sensory and Motor Neurons, J Neurosc 22 (2002), 3512-3519.

[13] A.D. Cohen, J.L. Tillerson, A.D. Smith, T. Schallert and M.J. Zigmond, Neuroprotective effects of prior limb use in 6hydrxydopamine-treated rats: possible role of GDNF, $J$ Neurochemistry 85 (2003), 299-305.

[14] P. Cordo, V.S. Gurfinkel, L. Bevan and G.K. Kerr, Proprioceptive consequences of tendon vibration during movement, $J$ Neurophysiol 74 (1995), 1675-1688.

[15] R. de la Fuente-Fernandez, T.J. Ruth, V. Sossi, M. Schulzer, D.B. Calne and A.J. Stoessl, Expectation and Dopamine Release: Mechanism of the Placebo Effect in Parkinson's disease, Science 293 (2000), 1164-1166.

[16] R. de la Fuente-Fernandez, M. Schulzer and A.J. Stoessl, The placebo effect in neurological disorders, Lancet Neurol 1 (2002), 85-91.

[17] S. Fahn, Is levodopa toxic? Neurology 47(Suppl 3) (1996), 184-195.

[18] J.M. Fearbly and A.J. Lees, Aging and Parkinson's disease: substantial nigra regional selectivity, Brain 114 (1991), 22832301.

[19] T. Foltynie, C. Brayne and R.A. Baker, The heterogeneity of idiopathic Parkinson's disease, J Neurol 249 (2002), 138-145.

[20] M.J. Griffin, Handbook of Human Vibration, San Diego, Academic press, 1996.

[21] C.G. Goetz, S. Leurgans, R. Raman and G.T. Stebbins, Objective changes in motor function during placebo treatment in PD, Neurology 54 (2000), 710-714.

[22] C.G. Goetz, S. Leurgans and R. Raman, and the Parkinson Study Group, Placebo-Associated Improvements in Motor Function: Comparison of Subjective and Objective Sections of the UPDRS in Early Parkinson's Disease, Mov Disord 17(2) (2002), 283-288.

[23] C.T. Haas, S. Turbanski and D. Schmidtbleicher, Postural control training in Parkinson's disease, Isok Ex Sci 12 (2004), 12-13.

[24] C.T. Haas, S. Turbanski and D. Schmidtbleicher, Effects of whole-body vibration on postural control in Parkinson's disease, Mov Disord 19(Suppl. 9) (2004), 185, P518.

[25] C.T. Haas, Simulation und Regulation Mechanischer Schwingungen im Alpinen Skirennlauf, Köln, Sport \& Buch Strauss, 2002.

[26] C.T. Haas, S. Turbanski, I. Kaiser and D. Schmidtbleicher, Biomechanische und physiologische Effekte mechanischer Schwingungsreize beim Menschen, Dt Zeitsch Sportmed 55 (2004), 34-43.

[27] K.E. Hagbarth and G. Eklund, Tonic vibration reflex (TVR) in spasticity, Brain Res 2 (1966), 201-203.

[28] P. Hobson, A. Holden and J. Meara, Measuring the impact of Parkinson's disease with the Parkinson's Disease Quality of Life questionnaire, Age and Aging 28(4) (1999), 341-346.

[29] Y.P. Ivanenko, V.L. Talis and O.V. Kazennikov, Support stability influences postural responses to muscle vibration in humans, Eur J Neurosc 11 (1999), 647-654.

[30] Y.P. Ivanenko, R. Grasso and F. Lacquaniti, Neck muscle vibration makes walking humans accelerate in the direction of gaze, J Physiol 525 (2000), 803-814. 
[31] Y.P. Ivanenko, R. Grasso and F. Lacquaniti, Influence of leg muscle vibration on human walking, J Neurophysiol $\mathbf{8 4}$ (2000), 1737-1747.

[32] M. Jahanshahi, I.H. Jenkins, R.G. Brown, C.D. Marsden, R.E. Passingham and D.J. Brooks, Self-initiated versus externally triggered movements I. An investigation using measurement of regional cerebral blood flow with PET and movement-related potentials in normal and Parkinson's disease subjects, Brain 118(4) (1995), 913-933.

[33] J. Jankovic, Levodopa strength and weakness, Neurology 58(Suppl. 1) (2002), 19-32.

[34] I.H. Jenkins, M. Jahanshahi, M. Jueptner, R.E. Passingham and D.J. Brooks, Self-initiated versus externally triggered movements II. The effect of movement predictability on regional cerebral blood flow, Brain 123 (2000), 1216-1228.

[35] E.M. Jöbges, J. Elek, J.D. Rollnik, R. Dengler and W. Wolf, Vibratory proprioceptive stimulation affects Parkinson tremor, Parkinsonism Relat Disord 8 (2002), 171-176.

[36] M. Jueptner, K.M. Stephan, C.D. Frith, D.J. Brooks, R.S.J. Frackowiak and R.E. Passingham, Anatomy of Motor Learning. I. Frontal Cortex and Attention to Action, J Neurophysiol 77 (1997), 1313-1324.

[37] M. Jueptner, C.D. Frith, D.J. Brooks, R.S.J Frackowiak and R.E. Passingham, Anatomy of Motor Learning. II. Subcortical structures and learning by Trail and Error Frontal Cortex and Attention to Action, J Neurophysiol 77 (1997), 1325-1337.

[38] K.H. Karlsen, E. Tandberg, D. Arsland and J.P. Larsen, Health related quality of life in Parkinson's Disease: a prospective longitudinal study, J Neurol Neurosurg Psychiatry 69(5) (2000), 584-589.

[39] T. Kasai, M. Kawanishi and S. Yahagi, The effects of wrist muscle vibration on human voluntary elbow flexion-extension movements, Exp Brain Res 90 (1992), 217-220.

[40] A. Kavounoudias, J.C. Gilhodes, R. Roll and J.P. Roll, From balance regulation to body orientation: two goals for muscle proprioceptive information processing? Exp Brain Res 124 (1999), 80-88.

[41] A. Kavounoudias, R. Roll and J.P. Roll, The plantar sole is a "dynamometric map" for human balance control, Neurorep 9(14) (1998), 3247-3252.

[42] A. Kavounoudias, R. Roll and J.P. Roll, Specific whole-body shifts inducted by frequency-modulated vibrations of human plantar soles, Neuroscience Letters 266(3) (1999), 181-184.

[43] E. Khudados, F.W.J. Cody and D.J. O'Boyle, Proprioceptive regulation of voluntary ankle movements, demonstrated using muscle vibration is impaired by Parkinson's disease, J Neurol Neurosurg Psychiatry 67 (1999), 504-510.

[44] J. Mally and T.W. Stone, Improvement of parkinsonian symptoms after repetitive transcranial magnetic stimulation, $\mathrm{J} \mathrm{Neu}$ rol Sci 162 (1999), 179-184.

[45] J. Mally and T.W. Stone, Therapeutic and "dose dependent" effect of repetitive transcranial magnetic stimulation, J Neurol Res 57 (1999), 935-940.

[46] C.D. Marsden, Fluctuations of disability in PD. Clinical aspects, in: Movement Disorders, C.D. Marsden and S. Fahn, eds, London, Butterworth scientific, 1981, pp. 96-122.

[47] P.B. Matthews, The reflex excitation of the soleus muscle of the decebrate cat caused by vibration applied to wrist tendon, J Physiol 184 (1966), 450-472.

[48] C. Maurer, T. Mergner, J. Xie, M. Faist, P. Pollak and C.H. Lücking, Effect of chronic bilateral subthalamic nucleus (STN) stimulation on postural control in Parkinson's disease, Brain 126 (2003), 1146-1163.
[49] G.E. McCall, R.E. Grindeland, R.R. Roy and V.R. Edgerton, Muscle afferent activity modulates bioassayable growth hormone in human plasma, J Appl Physiol 89 (2000), 1137-1141.

[50] E. Naito, T. Kochiyama, R. Kitatda, S. Nakamura, M. Michikazu, Y. Yonekura and N. Sadato, Internally Simulated Movement Sensations during Motor Imagery Activate Cortical Motor Areas and the Cerebellum, J Neurosc 22 (2002), 3683-3691.

[51] H. Nakamura, T. Moroji, S. Nohara, H. Nakamura and A. Okada, Activation of cerebral dopaminergic systems by noise and whole-body vibration, Environ Res 57 (1992), 10-18.

[52] H. Nakamura, T. Moroji, H. Nagase, T. Okazawa and A. Okada, Changes of cerebral vasoactive intestinal polypeptideand somatostatin-like immunoreactivity induced by noise and whole-body vibration in the rat, Eur J Appl Physiol Occup Physiol 68 (1994), 62-67.

[53] A.J. Nelson, W.R. Staines and W.E. McIlroy, Tactile stimulus predictability modulates activity in a tactile-motor cortical network, Exp Brain Res 154 (2004), 22-32.

[54] T. Ohnishi, T. Hayashi, S. Okabe, H. Matsuda, H. Iida and Y. Ugawa, Endogenous dopamine release by repetitive transcranial magnetic stimulation over the primary motor cortex, Mov Disord 19(Suppl. 9) (2004), 28.

[55] P. O'Suilleabhain, J. Bullard and R.B. Dewey, Proprioception in Parkinson's disease is acutely depressed by dopaminergic medication, J Neurol Neurosurg Psychiatry 71 (2001), 607610 .

[56] E.D. Playford, I.H. Jenkins, R.E. Passingham, J. Nutt, R.S. Frackowiak and D.J. Brooks, Impaired medial frontal and putamen activation in Parkinson's Disease: a positronen emission tomography study, Ann Neurol 32 (1992), 151-161.

[57] C. Rickards and F.W.J. Cody, Proprioceptive control of wrist movements in Parkinson's disease, Brain 120 (1997), 977990.

[58] J. Rowe, K.E. Stephan, K. Friston, R. Frackowiak, A. Lees and R. Passingham, Attention to action in Parkinson's disease. Impaired effective connectivity among frontal cortical regions, Brain 125 (2002), 276-289.

[59] U. Sabatini, K. Boulanouar, N. Fabre, F. Martin, C. Carel, C. Colonnese, L. Bozzao, I. Berry, J.L. Montastruc, F. Chollet and O. Rascol, Cortical motor reorganisation in akinetic patients with Parkinson's Disease. A functional MRI study, Brain 123 (2000), 394-403

[60] W.I. Schöllhorn, Individualität - ein vernachlässigter Parameter? Leistungssport 5 (1999), 5-12.

[61] A. Schrag, M. Jahanashahi and N. Quinn, What contributes to quality of life in patients with Parkinson's disease? J Neurol Neurosurg Psychiatry 69 (2000), 308-312.

[62] W. Schultz, Predictive reward signal of dopamine neurons, $J$ Neurophys 80 (1998), 1-27.

[63] W. Schultz, P. Dayan and R.R. Montague, A neural substrate of prediction and reward, Science 275 (1997), 1593-1599.

[64] J. Sian, M. Gerlach, M.B.H. Youdim and P. Riederer, Parkinson's Disease: a major hypokinetic basalganglia disorder, $J$ Neural Transm 106 (1999), 443-476.

[65] H.R. Siebener, C. Mentschel, C. Auer and B. Conrad, Repetitive transcranial magnetic stimulation has a beneficial effect of on bradykinesia in Parkinson's Disease, Neuroreport 10 (1999), 589-594.

[66] H.R. Siebener, C. Rossmeier, C. Mentschel, A. Peinemann and B. Conrad, Short term motor improvements after subthreshold repetitive transcranial magnetic stimulation of the primary motor hand area in Parkinson's Disease, J Neurol Sci 178 (2000), 91-94. 
[67] M. Steyvers, O. Levin, S.M. Verschueren and S.P. Swinnen, Frequency-dependent effects of muscle tendon vibration on corticospinal excitability: a TMS study, Exp Brain Res 151(1) (2003), 9-14.

[68] J.L. Tillerson, A.D. Cohen, W.M. Caudle, M.J. Zigmond, T. Schallert and G.W. Miller, Forced Nonuse in Unilateral Parkinsonian Rats Exacerbates Injury, J Neurosc 22 (2002), 67906799.

[69] S. Toledo-Frankel, N. Giladi, L. Gruendlinger, R. Baltadjieva, T. Herman and J.M. Hausdorf, Treadmill walking as an external cue to improve gait rhythm and stability in Parkinson's disease, Mov Disord 19(Suppl. 9) (2004), 138, 376.
[70] S.M.P. Verschueren, S.P. Swinnen, P.J. Cordo and N.V. Dounskaia, Proprioceptive control of multijoint movement: unimanual circle drawing, Exp Brain Res 121 (1999), 171-181.

[71] S.M.P. Verschueren, S.P. Swinnen, P.J. Cordo and N.V. Dounskaia, Proprioceptive control of multijoint movement: bimanual circle drawing, Exp Brain Res 121 (1999), 182-192.

[72] S.M.P. Verschueren, S.P. Swinnen, K. Desloovere and J. Duysens, Vibration-induced changes in EMG during Human Locomotion, J Neurophysiol 89 (2003), 1299-1307.

[73] M.M. Wierzbicka, J.C. Gilhodes and J.P. Roll, VibrationInduced Postural Posteffects, J Neurophysiol 79 (1998), 143150 . 